\title{
Fellow or foe: the impact of fellowship training programs on the education of Canadian urology residents
}

\author{
Ethan D. Grober, MD, MEd; ${ }^{*}$ Dean S. Elterman, MD; ${ }^{\dagger}$ Michael A.S. Jewett, $M D^{\ddagger}$
}

See related article on page 38

\begin{abstract}
Objective: Throughout North America, increasing emphasis is being placed on surgical fellowships. Surgical educators and trainees have raised concerns that the escalating focus on fellowships may threaten the educational mission of more novice trainees. Our objective was to collect opinions from multiple perspectives (faculty, fellows and residents) regarding fellowship structure, fellow selection and the impact of clinical fellowships on urology resident training.
\end{abstract}

Methods: We anonymously surveyed 52 members of a major academic urology training program (University of Toronto) with established fellowship training programs for their opinions regarding fellowship structure, fellow selection, and the impact on resident training and education.

Results: The overall response rate was $88 \%$. We identified significant differences of opinion among faculty, fellows and residents regarding fellowship structure, fellow selection and the impact on resident education. Specifically, faculty and fellows supported the addition of more fellows, felt that certain complex cases should be designated as "fellow cases" and that residents' research opportunities were not restricted. Residents felt that fellows "steal" operative cases, that performing operations with the fellow is not equivalent to performing operations with faculty alone and that fellowship candidates should perform an operation with division faculty as part of the application process. There was agreement that fellowship programs add value to residents' overall education, that fellows should participate in the call schedule and that fellows' role in the operating room needs to be better defined with respect to case volume and selection. Proficiency in technical skills, clinical knowledge, teaching and teamwork were cited as the most attractive characteristics of an effective clinical fellow.

Conclusion: Residency and fellowship program directors must clearly define the role of the fellow and outline the limits of surgical practice, establish clear and consistent guidelines outlining responsibilities (operative, clinical and on-call), and open lines of communication to ensure that all opinions are recognized and addressed. Finally, they must select fellows with proficient technical skills, clinical knowledge, teaching ability and work ethic to ensure that they focus on "specialized" training.

CUAJ 2008;2(1):33-7

\section{Introduction}

Over the past decade, medicine has undergone significant evolution and has witnessed a phenomenal growth of knowledge and innovative technology. ${ }^{1-3}$ In urological surgery, the technological revolution that began in the late 1980s with introduction of laparoscopic surgery has given birth to a variety of technology-driven and minimally invasive operations, many of which have evolved to become the "standard of care." ${ }^{\prime 4-6}$

In the face of such rapid and widespread acceptance of new technologies, coupled with a forever-expanding medical curriculum, issues concerning education and training have become paramount. Longer and more advanced training programs with increased specialization for urological surgeons have emerged as coping strategies in both education and practice.

Throughout the urological community in Canada and the United States, increasing emphasis is being placed on surgical fellowships that provide periods of highly specialized and advanced surgical training, following the completion of formal residency training. Currently, the Endourological Society recognizes 53 clinical fellowship programs ${ }^{7}$; the Society for Pediatric Urology endorses 23 fellowships ${ }^{8}$; and the Society of Urologic Oncology has 16 accredited fellowship training programs. ${ }^{9}$

Fellows offer several potential benefits to the surgical department and faculty. They contribute to the academic and financial productivity of the department, provide a high level of clinical service to patients, teach residents and medical students, share in the on-call duties, and represent a vehicle for national and international dissemination of program expertise and reputation.

Increasingly, surgical educators have raised concerns that the escalating focus on fellowship training may threaten the basic educational mission of academic training programs and limit the experience of more 
novice learners, including surgical residents and medical students. ${ }^{10,11}$ In a recent conversation about medical education, the president of Johns Hopkins University recounted a discussion with a professor at his institution. When queried about the priorities for medical student education, the essence of the professor's response was revealed in his use of possessive pronouns: "my fellows, our residents, your medical students." 12

Recent surveys of residents from major academic surgical training centres with well-developed fellowship structures identified concerns that the increasing presence of fellows and designation of complex surgeries as "fellow cases" will translate into less surgical volume and fewer opportunities for meaningful participation in the operating room..$^{13}$ Diluting the surgical experience of residents may perpetuate feelings of unpreparedness for independent clinical practice and result in conflict and tension between residents and fellows.

With the overall goal to enhance program quality for the urological faculty, fellows and residents, the primary objective of our study was to highlight opinions from multiple perspectives (i.e., faculty, fellows and residents) regarding fellowship structure, fellow selection (i.e., number and qualities) and the impact of clinical fellowships on urology resident training and education.

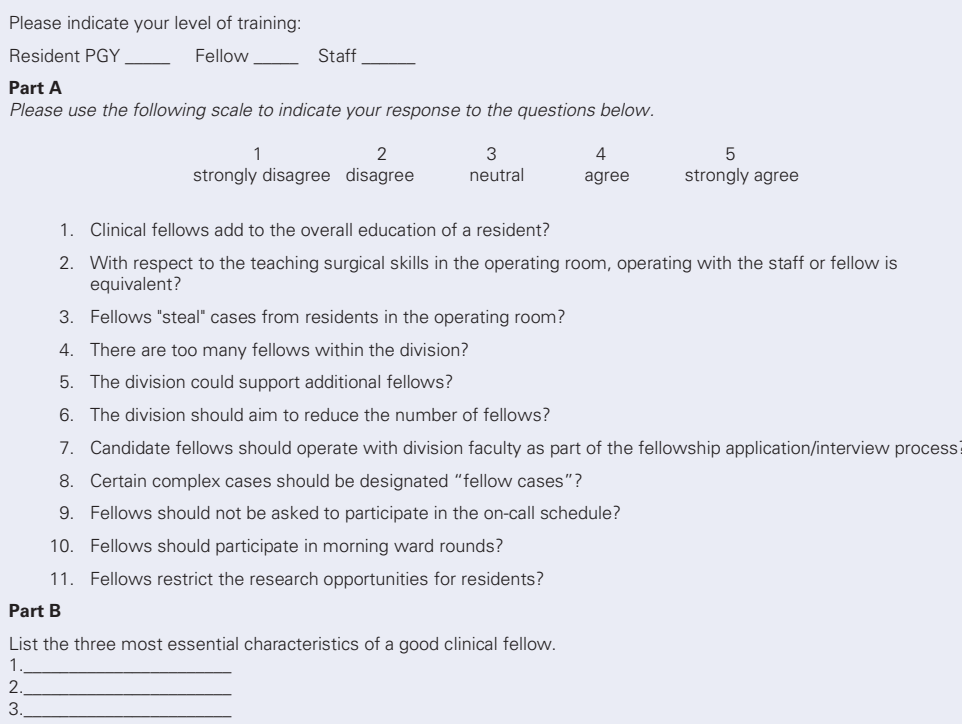

Fig. 1. Example of a fellowship survey.

\section{Methods}

During the 2005/06 academic year, members of the Division of Urology at the University of Toronto (22 faculty members, 13 fellows and 17 residents) were voluntarily and anonymously administered a 12-question survey designed to highlight attitudes regarding fellowship structure, fellow selection, and the impact on resident training and education (Fig. 1). Informed consent was obtained before participation in the study. Statistics were analyzed using SPSS, version 12.0 (SPSS Inc., Chicago, Illinois). Analysis of variance (ANOVA) was used to compare mean survey scores between groups.

The Division of Urology at the University of Toronto was selected as the "study" program because it has a mature, highly competitive (national and international), well-established fellowship structure in various subspecialties (e.g., trauma and reconstruction, female urology, neurourology, male infertility, oncology, pediatric urology, endourology and laparoscopic surgery), at multiple hospital sites where faculty, fellows, residents and medical students interact with a shared population of patients.

Over the past decade, the number of clinically active surgical fellows within the University of Toronto's Department of Surgery has grown from 101 in $1993 / 94$ to 113 in 1998/99 to 165 in 2003/04.

\section{Results}

The overall response rate was $88 \%$ (faculty $18 / 22$, fellows $11 / 13$, residents $17 / 17)$. We identified

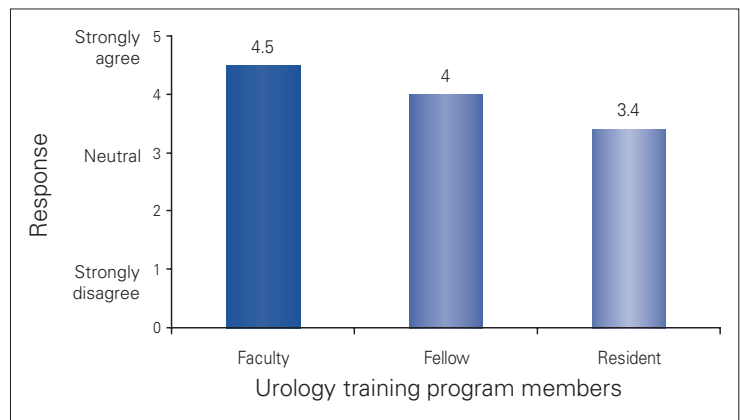

Fig. 2. Survey response to whether "clinical fellows add to the education of a resident?" In contrast with residents, faculty and fellows expressed stronger feelings about the value of fellows to resident education. Note: $p<0.001$ faculty v. fellow; $p=0.04$ fellow v. resident; $p<0.001$ overall. 
significant differences between faculty, fellow and resident opinions regarding fellowship structure, fellow selection and the impact on resident education. Survey responses are graphically depicted

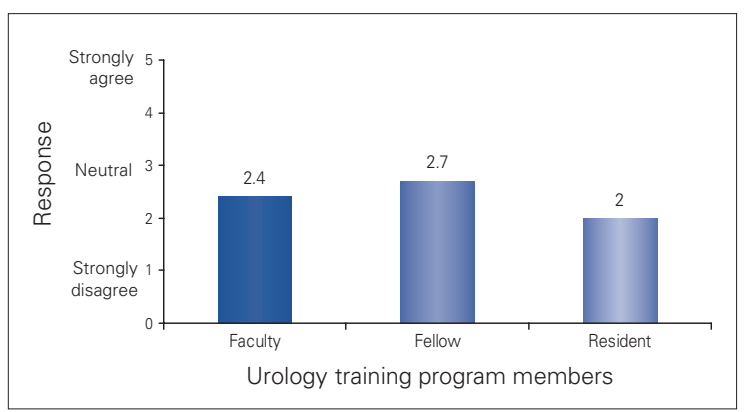

Fig. 3. Survey response to whether "with respect to teaching surgical skills in the $O R$, operating with the staff or fellow is equivalent?" In contrast with fellows, residents felt that performing operations with the fellow was not equivalent to operating with faculty. Note: $p=0.04$ fellow v. resident; $p=0.05$ overall. $\mathrm{OR}=$ operating room.

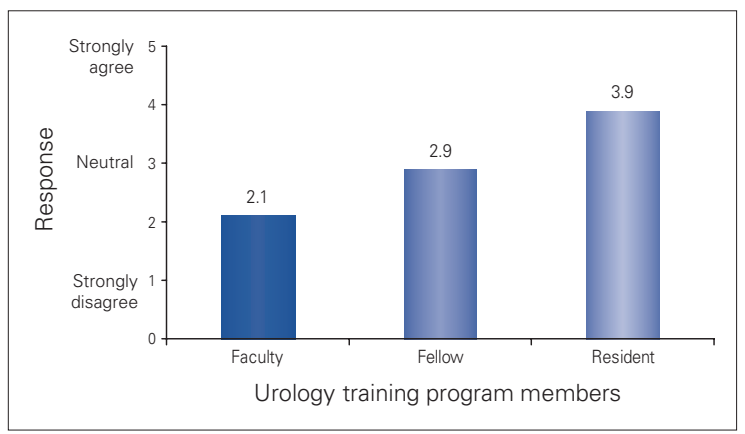

Fig. 5. Survey response to whether "there are too many fellows within the division?" Compared with faculty and fellows, residents felt that there were too many fellows within the division of urology. Note: $p<0.001$ faculty v. resident; $p=0.01$ faculty v. fellow; $p=0.001$ fellow $v$. resident; $p<0.001$ overall.

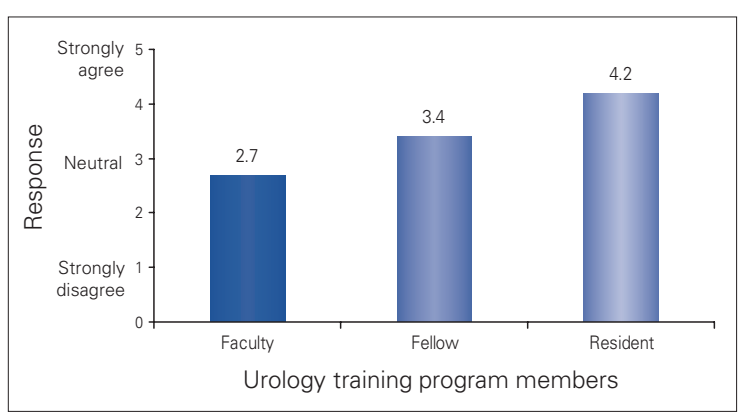

Fig. 7. Survey response to whether "candidate fellows should operate with division faculty as part of the fellowship application/interview process?" In contrast with faculty, residents felt that the technical skills of fellows should be formally evaluated before program acceptance. Note: $p<0.001$ faculty v. resident; $p<0.001$ overall. in Figure 2, Figure 3, Figure 4, Figure 5, Figure 6, Figure 7, Figure 8, Figure 9, Figure 10 and Figure 11.

Proficient technical skills, teaching ability, teamwork and clinical knowledge were cited as the

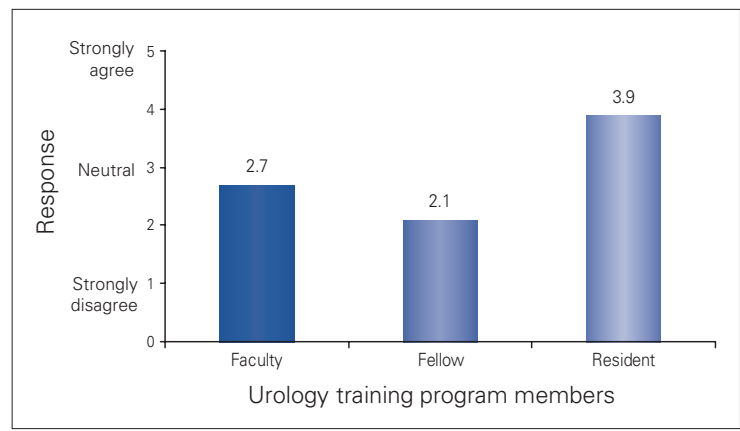

Fig. 4. Survey response to whether "fellows 'steal' cases from residents in the OR?" In contrast with residents, faculty and fellows felt that fellows did not "steal" cases in the operating room. Note: $p<0.001$ faculty v. resident; $p<0.001$ fellow v. resident; $p<0.001$ overall. $\mathrm{OR}=0$ operating room.

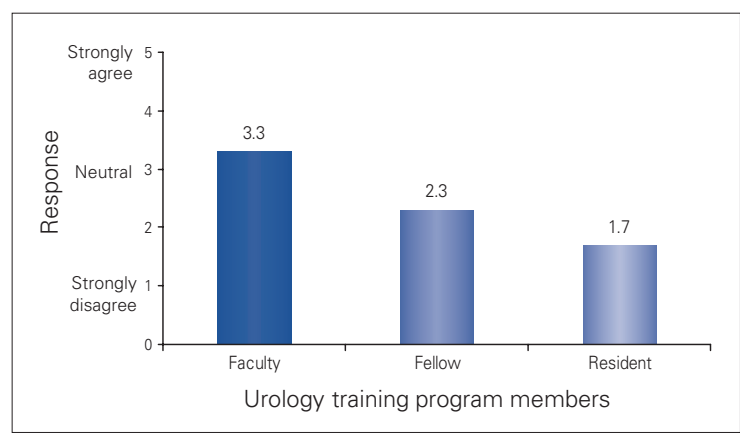

Fig. 6. Survey response to whether "the division could support additional fellows?" In contrast with residents and fellows, faculty felt the division could support additional fellows. Note: $p<0.001$ faculty v. resident; $p=0.002$ faculty v. fellow; $p<0.001$ overall.

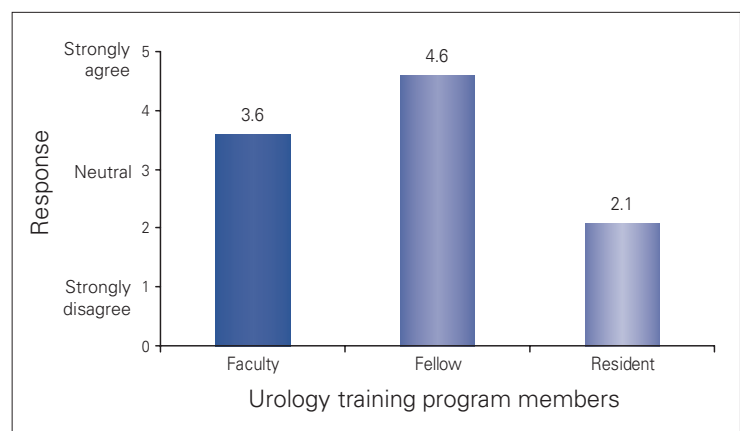

Fig. 8. Survey response to whether "certain complex cases should be designated 'fellow cases'?" In contrast with residents, faculty and fellows felt that certain complex cases should be designated as "fellow cases." Note: $p=0.002$ faculty v. resident; $p<0.001$ fellow v. resident; $p<0.001$ overall. 
most essential characteristics of an effective clinical fellow (Fig. 12).

\section{Discussion}

Based on the results of this survey, we identified significant differences of opinion between urological faculty, fellows and residents regarding fellowship structure, fellow selection and the impact on resident education. Specifically, faculty and fellows felt the number of fellows was appropriate and they supported the addition of more fellows. Additionally, they felt that certain complex cases should be designated as "fellow cases" and residents' research opportunities were not restricted. Residents felt that fellows "steal" operative cases, that performing operations with the fellow is not equivalent to performing operations with faculty alone and that fellowship candidates should operate with division faculty as part of the application process. There was agreement that

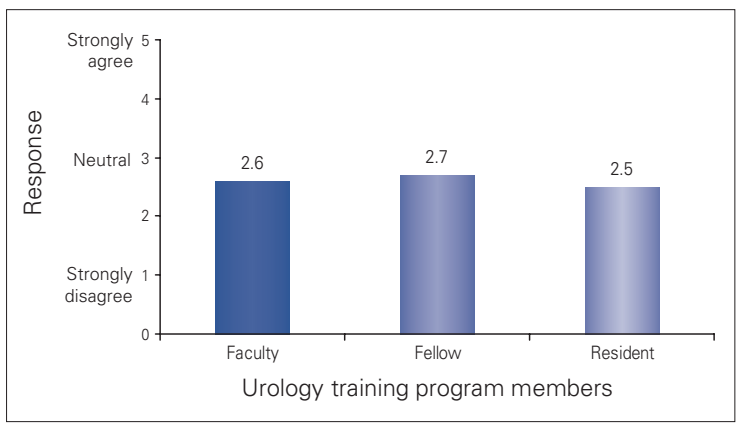

Fig. 9. Survey response to whether "fellows should NOT participate in the on-call schedule?" Faculty, fellows and residents were neutral on whether fellows should participate in the on-call schedule. Note: $p=0.8$ overall.

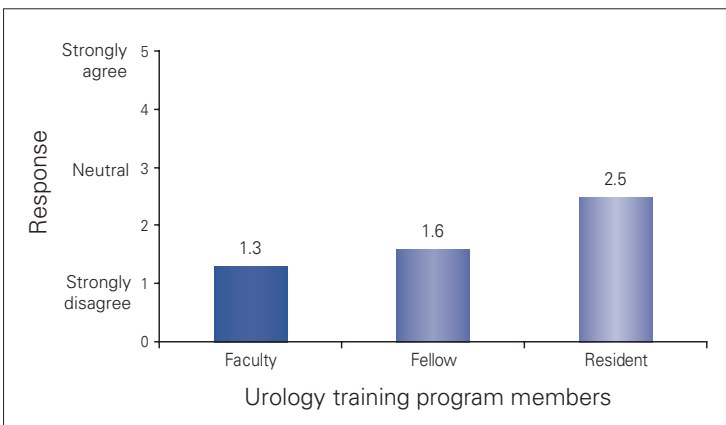

Fig. 11. Survey response to whether "fellows restrict the research opportunities for residents?" In contrast with residents, faculty and fellows did not feel that fellows restricted the research opportunities for residents. Note: $p=0.001$ faculty v. resident; $p<0.02$ fellow $v$. resident; $p=0.001$ overall. fellowship programs add value to the overall education of residents, that fellows should participate in the on-call schedule and that the role of fellow in the operating room needs to be better defined with respect to case volume and selection. Proficiency in technical skills, clinical knowledge, teaching and teamwork were cited as the most attractive characteristics of an effective clinical fellow.

We recognize that this data highlights the opinions of members of a surgical division from a single centre and that regional, national and international differences based on program philosophy and structure likely exist. However, our article is the first to address this issue in the urological literature and we hope our findings will stimulate further discussion and future scholarly investigation. As pressures from licensing authorities to track

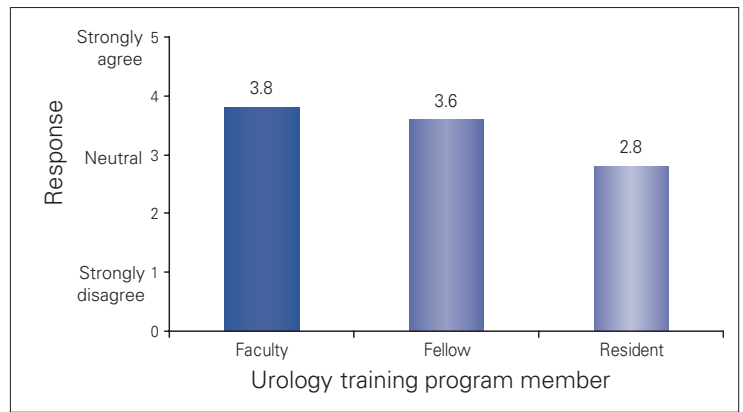

Fig. 10. Survey response to whether "fellows should participate in ward rounds?" In contrast with residents, faculty felt that fellows should participate in patient ward rounds. Note: $p=0.009$ faculty v. resident; $p<0.009$ overall.

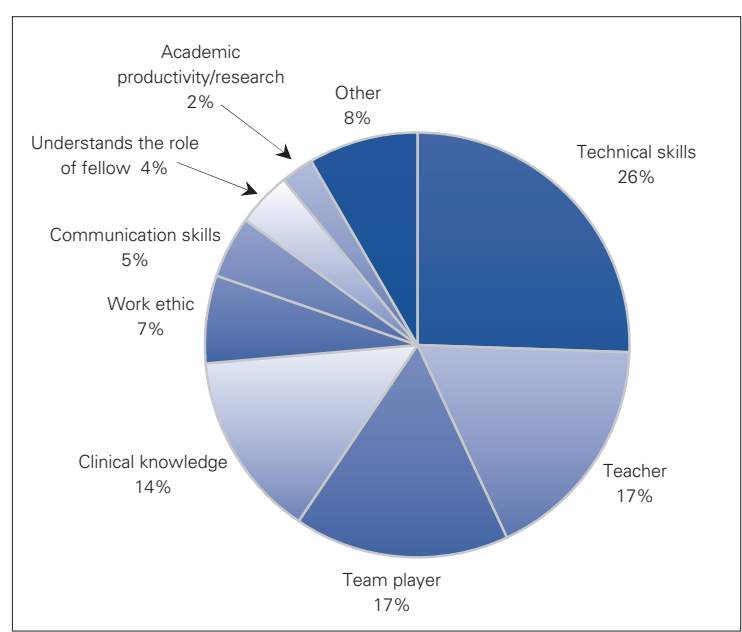

Fig. 12. Survey response to "essential characteristics of a fellow?" Proficiency in technical skills, teaching and teamwork were most commonly cited essential characteristics of good clinical fellows. 
case volumes and surgical participation increase, the opinions reported in this paper can be validated with objective data.

As fellowship training programs continue to expand in all domains of urology, academic surgeons must become increasingly sensitive to the perceived impact that fellows have on the educational experience of surgical trainees and not lose sight of the ultimate goals of residency training:

- "To ensure that residents are competent in the diagnosis and medical and surgical management of a wide variety of urologic disorders"14 (American Urological Association).

- "To ensure that each graduate physician will be competent to function as a consultant surgical specialist"15 (Royal College of Physicians and Surgeons of Canada).

In an effort to circumvent internal conflict and promote a mutually beneficial working atmosphere, residency and fellowship program directors must clearly define the role of the surgical fellow and outline the limits of surgical practice within the philosophy of the existing residency program.

Even in the face of an increasingly specialized global impact health care system, academic teaching centres must strengthen their commitment to educating the physicians of the future - at all levels. It is our hope that through such a commitment, in the very near future, professors and faculty will refer to their trainees as "my fellows, my residents and my medical students."

\section{Conclusion}

Based on the results of the current study, we offer the following recommendations to academic urology programs that have both residents and fellows. Residency and fellowship program directors must

1. clearly define the role of the fellow and outline the goals and limits of surgical practice within the philosophy of the existing residency program for both residents and fellows;

2. establish clear, consistent guidelines outlining operative, clinical and on-call responsibilities;

3. ensure open lines of communication between faculty, fellows and residents;

4. ensure the selection of fellows with proficient technical skills, clinical knowledge, teaching ability and work ethic, so they focus on "specialized" training versus training in "basic" urological surgery;

5. base the number of clinical fellows on hospital site case volumes, not on the need to fill a temporary void in resident numbers;

6. ensure residents continue to take an active role in complex, innovative and technology-driven procedures; and

7. share and learn from the experiences of other surgical programs.

From the *Division of Urology, Mount Sinai Hospital and Women's College Hospital, University of Toronto, Toronto, Ont., the †Division of Urology, University of Toronto, Toronto, Ont., and the ‡Division of Urology, Department of Surgical Oncology, Princess Margaret Hospital and the University Health Network, University of Toronto, Toronto, Ont.

This article has been peer reviewed.

Competing interests: None declared.

\section{References}

1. Hemal AK, Menon M. Robotics in urology. Curr Opin Urol 2004;14:89-93.

2. Reznick RK. Virtual reality surgical simulators: Feasible but valid? J Am Coll Surg 1999; 189:127-8.

3. Grober ED, Hamstra SJ, Wanzel KR, et al. Laboratory based training in urological microsurgery with bench model simulators: a randomized controlled trial evaluating the durability of technical skill. J Urol 2004;172:378-81.

4. Sain AH. Laparoscopic cholecystectomy is the current "gold standard" for the treatment of gallstone disease. Ann Surg 1996;224:689-90.

5. Gill IS. Laparoscopic radical nephrectomy for cancer. Urol Clin North Am 2000;27:707-19.

6. Rubinstein $M$, Gill IS, Aron $M$, et al. Prospective, randomized comparison of transperitoneal versus retroperitoneal laparoscopic adrenalectomy. J Urol 2005; 174:442-5.

7. Endourological Society. Recognized fellowship programs. Available: www .endourology.org/fellowship/index.html (accessed 2008 Jan 3).

8. The Society for Pediatric Urology. Participating fellowship programs. Available: www .spuonline.org/fellowships_pp.cgi (accessed 2008 Jan 3).

9. Society of Urologic Oncology. Approved fellowship programs. Available: www .societyofurologiconcology.org/fellowships/ (accessed 2008 Jan 3).

10. Allen WC. The relationship between residency programs and fellowships in the education setting. Clin Orthop Relat Res 1990;257:57-60.

11. Minor S, Poenaru D. The in-house education of clinical clerks in surgery and the role of housestaff. Am I Surg 2002;184:471-5.

12. Brody WR. My fellows, my students. Johns Hopkins Medicine. Crossroads: essays and columns. Available at: www.hopkinsmedicine.org/about/crossroads (accessed 2008 Jan 3).

13. Surgical Spotlight. Department of Surgery - University of Toronto Magazine. May 2006; $1-3$.

14. The American Urological Association. Guidelines to urology residency programs. Available: www.auanet.org/residents/whatisurology.cfm (accessed 2008 Jan 3).

15. The Royal College of Physicans and Surgeons of Canada. Examinations and credentials. Available: hittp://rrpsc.medical.org/residency/certification/index.php?page_title $=$ Examinations (accessed 2008 Jan 3).

Correspondence: Dr. Ethan D. Grober, Murray Koffler Urologic Wellness Centre, Mt. Sinai Hospital, Joseph and Wolf Lebovic Building, 60 Murray St., 6th Floor, Box 19, Toronto ON M5T 3L9; ethan.grober@utoronto.ca 